\title{
Quit smoking and reduce surgical complications
}

\author{
Jon Karlsson
}

Published online: 11 January 2011

(C) Springer-Verlag 2011

In this issue of the journal, Meidinger et al. [2] publish a paper with the title "May smokers and overweight patients be treated with a medial open-wedge HTO? Risk factors for non-union". This work is timely, and in fact, it is somewhat surprising that researchers involved in sports traumatology have not highlighted the risks of smoking when it comes to soft-tissue and bone healing and postsurgical complications. These researchers studied 182 patients, who were operated on with medial open-wedge high tibial osteotomy (HTO). The non-union rate was $5.4 \%$, which can be considered as a reasonably low number. However, when their patients are studied in more detail, they report that $7 / 10$ patients who suffered from non-union were smokers. In other words, a substantially increased risk of post-surgical complication and revision surgery was seen in smokers in this study. Their conclusion is that the factors; smoking and overweight/obesity played a significant role in the development of a non-union in patients treated with an open-wedge HTO. Similar observations have been reported in patients undergoing hip or knee replacement surgery.

Recently, several researchers have recommended smoking cessation prior to elective orthopaedic surgery. Nåsell et al. [3] assessed a smoking cessation program that was initiated during acute hospitalization period in patients suffering from acute fracture of the lower or upper extremity. The program was continued for 6 post-operative weeks. Altogether 105 patients were randomly allocated to an intervention group $(n=55)$ or a control group

J. Karlsson $(\bowtie)$

Department of Orthopaedics,

Sahlgrenska University Hospital,

Gothenburg, Sweden

e-mail: Jon.karlsson@telia.com
( $n=50)$. The results strongly indicated that a smoking cessation program during the first weeks after acute fracture surgery reduced the risk of post-operative complications. The proportion of patients with at least one post-operative complication was $20 \%$ in the smoking cessation group, compared with $38 \%$ in the control group. Lindström et al. [1] studied-also in a randomized trial-the effects of perioperative smoking cessation on post-operative complications in patients undergoing elective planned surgery. In that study, the intervention consisted of individual counselling and nicotine substitution that started 4 weeks prior to surgery and continued 4 weeks post-operatively. Altogether 117 patients were enrolled in this study. Overall complication rate in the control group was $41 \%$, compared with $21 \%$ in the intervention group. The relative risk reduction (RRR) for any post-operative complication was $49 \%$. The authors concluded that smoking cessation appeared to be an effective tool to reduce post-operative complications in patients undergoing planned surgery, even though the program was introduced as late as 4 weeks prior to surgery. Still another study by Villebro et al. [4] investigated the long-term effect (1-year follow-up) of smoking cessation. They randomly allocated 101 patients undergoing planned hip or knee replacement surgery to two groups; smoking intervention and no intervention. The patients were followed for 1 year after surgery. They found that significantly more patients in the intervention group abstained from smoking 1 year post-operatively ( 22 vs. $3 \%$ ). Five-year follow-up showed that $17 \%$ of the controls and $8 \%$ in the intervention group had died.

Now; what does all this mean? It is well known that smoking is a major health problem and moreover an economic concern. And, therefore, it is necessary to strongly underline benefits of smoking cessation in patients undergoing acute or planned orthopaedic surgery. The risk 
reduction of post-operative complications has been shown to be approximately $50 \%$. That is a lot.

All of us, who are involved in sports medicine and sports traumatology, especially when it comes to surgical interventions should take the opportunity to help our patients to quit smoking for better health and better economy.

\section{References}

1. Lindström D, Sadr Azodi O, Wladis A, Tönnesen H, Linder S, Nåsell H, Ponzer S, Adami J (2008) Effects of a perioperative smoking cessation intervention on postoperative complications. A randomized trial Ann Surg 248:739-745

2. Meidinger G, Imhoff AI, Paul J, Kirchoff C, Sauerschnig M, Hinterwimmer S (2011) May smokers and overweight patients be treated with a medial open-wedge HTO? Risk factors for nonunion. Knee surg Sports Traumatol Arthrosc 19. doi:10.1007/ s00167-010-1335

3. Nåsell H, Adami J, Samnegård E, Tönnersen H, Ponzer S (2010) Effect of smoking cessation intervention on results of acute fracture surgery: a randomized controlled trial. J Bone Joint Surg Am 92:1335-1342

4. Villebro NM, Pedersen T, Möller Am, Tönnesen H (2008) Longterm effects of a preoperative smoking cessation programme. Clin Respir J 2:175-182 